\title{
Colossal positive magnetoresistance in a doped nearly magnetic semiconductor
}

\author{
Rongwei Hu, ${ }^{1,2}$ K. J. Thomas, ${ }^{1, *}$ Y. Lee, ${ }^{3}$ T. Vogt, ${ }^{1, \dagger}$ E. S. Choi, ${ }^{4}$ V. F. Mitrović, ${ }^{2}$ R. P. Hermann, ${ }^{5, \$}$ F. Grandjean, ${ }^{5}$ \\ P. C. Canfield, ${ }^{6}$ J. W. Kim, ${ }^{6}$ A. I. Goldman, ${ }^{6}$ and C. Petrovic ${ }^{1}$ \\ ${ }^{1}$ Condensed Matter Physics, Brookhaven National Laboratory, Upton, New York 11973, USA \\ ${ }^{2}$ Physics Department, Brown University, Providence, Rhode Island 02912, USA \\ ${ }^{3}$ Department of Earth System Sciences, Yonsei University, Seoul 120749, Korea \\ ${ }^{4}$ NHMFL/Physics, Florida State University, Tallahassee, Florida 32310, USA \\ ${ }^{5}$ Department of Physics, Université de Liège, B-4000 Liège, Belgium \\ ${ }^{6}$ Ames Laboratory and Department of Physics and Astronomy, Iowa State University, Ames, Iowa 50011, USA \\ (Received 15 October 2007; revised manuscript received 6 December 2007; published 27 February 2008) \\ We report on a positive colossal magnetoresistance (MR) induced by metallization of $\mathrm{FeSb}_{2}$, a nearly \\ magnetic or "Kondo" semiconductor with $3 d$ ions. We discuss the contribution of orbital MR and quantum \\ interference to the enhanced magnetic field response of electrical resistivity.
}

DOI: $10.1103 /$ PhysRevB.77.085212

PACS number(s): 75.47.Gk, 71.30.+h, 72.15.Rn, 75.50.Pp

There is at present considerable technological interest in the magnetoresistive effect that is central to the operation of devices in magnetic storage media and a wide variety of magnetic sensors. The desire to maximize this effect has raised interest in new materials and mechanisms associated with the large change in electrical resistance in magnetic field. ${ }^{1} \mathrm{FeSb}_{2}$ is a narrow gap semiconductor whose magnetic properties strongly resemble nearly magnetic or "Kondo" insulator FeSi. ${ }^{2,3}$ The $a b$ initio local density approximation plus $U$ electronic calculations found that the ground state of $\mathrm{FeSb}_{2}$ is nearly ferromagnetic. ${ }^{4}$ Temperature induced paramagnetic moment for field applied parallel to $\hat{b}$ axis coincides with increased conduction at high temperatures. ${ }^{2,3,5}$ Electrical transport measurements showed pronounced anisotropy and metallic conductivity above $40 \mathrm{~K}$, and activated below that temperature for current applied along the $\hat{b}$ axis. The exact temperature of metal-semiconductor crossover was found to be very sensitive to small current misalignment, implying a quasi-one-dimensional nature of electronic transport. ${ }^{2}$ Optical conductivity revealed anisotropic energy gap $E_{g}$ in the spectral range between 100 and $350 \mathrm{~cm}^{-1}$ and negligible Drude weight of $\sigma(\omega)$ at low frequencies, i.e., a true insulating state. In addition, a full recovery of spectral weight occurs only above $1 \mathrm{eV}$, suggesting a contribution of larger energy scales than in conventional metal-insulator transitions where thermal excitations of charge carriers through $E_{g}$ brings about redistribution of the spectral weight just above the gap. ${ }^{6}$ Metal-insulator transitions in FeSi have been induced by band narrowing ${ }^{7}$ or by insertion of carriers, ${ }^{8}$ albeit with modest magnetoresistance. Unlike $\mathrm{Fe}_{1-x} \mathrm{Co}_{x} \mathrm{Si}$, colossal magnetoresistance (CMR) is observed for all $x \leqslant 0.4$ in $\mathrm{Fe}_{1-x} \mathrm{Co}_{x} \mathrm{Sb}_{2}$.

$\mathrm{Fe}_{1-x} \mathrm{Co}_{x} \mathrm{Sb}_{2}$ single crystals were grown from excess $\mathrm{Sb}$ flux. ${ }^{2} \mathrm{X}$-ray powder diffraction experiments and crystal orientation were performed at the beamlines X7A and X22C of the NSLS at the Brookhaven National Laboratory. Electrical transport and transverse magnetoresistance (MR) measurements were carried out in Quantum Design MPMS and PPMS instruments. For Hall resistivity $\rho_{x y}$, the current was applied along the highly conducting $\hat{b}$ axis and the voltage was picked up in the orthogonal (Hall) [101] direction. To completely cancel out the longitudinal voltage contribution, magnetic field was swept from $H=9 \mathrm{~T}$ to $H=-9 \mathrm{~T}$, and onehalf of the voltage difference was taken as the Hall voltage. The high field transverse MR measurements were carried out at the NHMFL in Tallahassee, FL up to $300 \mathrm{kOe}$. The powder X-ray patterns show that the Co substitution on the Fe site uniformly contracts the unit cell volume, in agreement with Vegard's law. ${ }^{5}$ The Mössbauer data indicate that there is no localized magnetism on the Fe site and no visible subspectrum of impurities.

The application of a magnetic field of $90 \mathrm{kOe}$ induces up to 2.5 orders of magnitude change in the resistivity in $\mathrm{Fe}_{1-x} \mathrm{Co}_{x} \mathrm{Sb}_{2}(x=0-0.4)$ and $30 \%$ of MR at room temperature for $x=0.1$ (Fig. 1). This is comparable to MR in the colossal magnetoresistive manganites. ${ }^{9}$ What could be the most likely cause of CMR in this system? The mechanism could involve a presence of charge carriers from different parts of the Fermi surface that have different scattering times or a breakdown of the semiclassical transport theory and relation between conductivity and scattering time $\rho \sim 1 / \tau{ }^{10}$

Hall resistivity is shown as a nonlinear function of magnetic field in Fig. 2(b). The pronounced field dependence of Hall constant is reminiscent of the anomalous Hall effect. When there are magnetic moments involved, the Hall resistivity can be written as ${ }^{11}$

$$
\rho_{x y}(H)=R_{0} H+R_{s} M(H),
$$

where $R_{0}$ and $R_{s}$ are the normal and spontaneous Hall constants, and $M$ is the sample magnetization. Fitting $\rho_{x y}$ data by using the experimental values of $M(H)$ [Fig. 2(a)], we found that the anomalous Hall effect is an unlikely cause of Hall resistivity nonlinearity. The nonlinearity of Hall resistivity in field and band structure calculations ${ }^{4}$ suggests that there are more than one band participating in the conduction (Fig. 3).

In what follows, we discuss the case when there are more than one type of carrier participating in the conduction. The Hall constant takes the general form ${ }^{12}$

$$
R_{H}=-\frac{1}{H} \frac{\sum \sigma_{x y}^{i}}{\left(\sum \sigma_{x x}^{i}\right)^{2}+\left(\sum \sigma_{x y}^{i}\right)^{2}}
$$



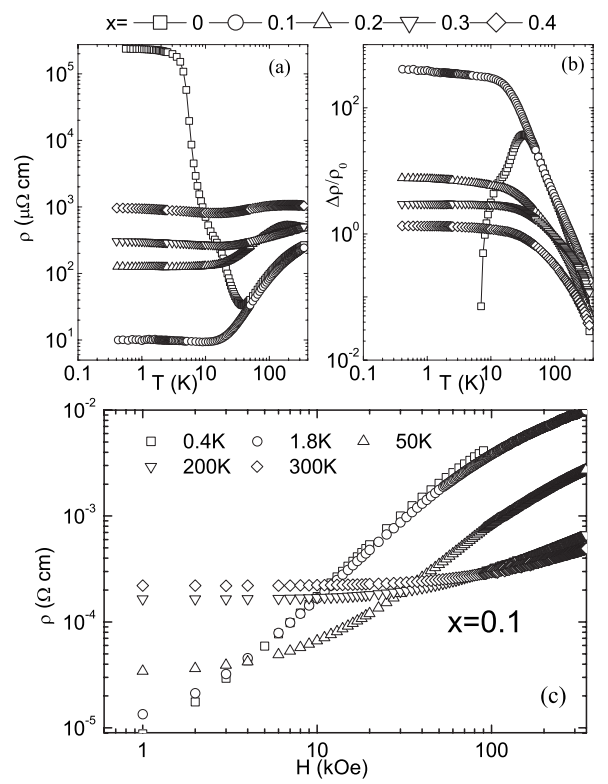

FIG. 1. (a) Electrical transport properties of $\mathrm{Fe}_{1-x} \mathrm{Co}_{x} \mathrm{Sb}_{2}$ dramatically change with a small change in shoichiometry. The sample with $x=0.1$ manifests the lowest resistivity. With further Co substitution, we see a gradual increase in residual resistivity. (b) Temperature dependence of magnetoresistance $\mathrm{MR}=[\rho(90 \mathrm{kOe})$ $-\rho(0)] / \rho(0)$ in doped $\mathrm{Fe}_{1-x} \mathrm{Co}_{x} \mathrm{Sb}_{2}$ semiconductor alloys for $x=0-0.4$. CMR is observed for all $x$. (c) Resistivity isotherms of $\mathrm{Fe}_{0.9} \mathrm{Co}_{0.1} \mathrm{Sb}_{2}$ up to $350 \mathrm{kOe}$. Resistivity increases 3 orders of magnitude $(103100 \%)$ at $1.8 \mathrm{~K}$.

$$
\sigma_{x x}^{i}=\frac{q n_{i} \mu_{i}}{1+\mu_{i}^{2} H^{2}}, \quad \sigma_{x y}^{i}=\frac{q n_{i} \mu_{i}^{2} H}{1+\mu_{i}^{2} H^{2}},
$$

where $\sigma_{x x}^{i}$ and $\sigma_{x y}^{i}$ are longitudinal and Hall conductivities of individual bands, and $\mu_{i}$ is the band mobility. A matrix formalism for the Hall effect in multicarrier semiconductor systems was devised and provided a closed form formula for two- or three-carrier systems. ${ }^{13,14}$ The magnetoresistance within the same formalism for a two-carrier system is

$$
\begin{gathered}
R_{H}=\rho_{0} \frac{\alpha_{2}+\beta_{2} H^{2}}{1+\beta_{3} H^{2}}, \quad M R_{D}=\frac{\alpha_{D} H^{2}}{1+\beta_{D} H^{2}}, \\
\alpha_{2}=f_{1} \mu_{1}+f_{2} \mu_{2}, \quad \beta_{2}=\left(f_{1} \mu_{2}+f_{2} \mu_{1}\right) \mu_{1} \mu_{2}, \\
\beta_{3}=\beta_{D}=\left(f_{1} \mu_{2}+f_{2} \mu_{1}\right)^{2}, \quad \alpha_{D}=f_{1} f_{2}\left(\mu_{1}-\mu_{2}\right)^{2},
\end{gathered}
$$

and for three-carrier systems,

$$
\begin{gathered}
R_{H}=\rho_{0} \frac{\alpha_{2}+\beta_{2} H^{2}+\gamma_{2} H^{4}}{1+\beta_{3} H^{2}+\gamma_{3} H^{4}}, \\
M R_{T}=\frac{\left(\alpha_{T}+\gamma_{T} H^{2}\right) H^{2}}{1+\left(\beta_{T}+\delta_{T} H^{2}\right) H^{2}}, \\
\alpha_{2}=f_{1} \mu_{1}+f_{2} \mu_{2}+f_{3} \mu_{3}, \\
\beta_{2}=f_{1} \mu_{1}\left(\mu_{2}^{2}+\mu_{3}^{2}\right)+f_{2} \mu_{2}\left(\mu_{1}^{2}+\mu_{3}^{2}\right)+f_{3} \mu_{3}\left(\mu_{1}^{2}+\mu_{2}^{2}\right),
\end{gathered}
$$
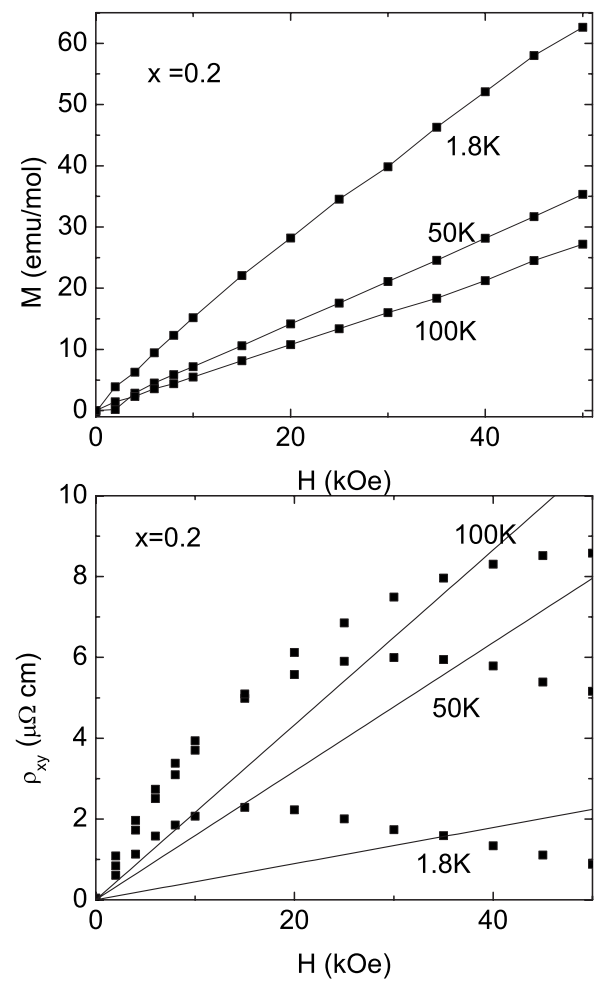

FIG. 2. Hall resistivity versus magnetic field. Solid lines are the fits when magnetization $M$ is taken into account.

$$
\begin{gathered}
\beta_{3}=\beta_{T}=\left(f_{1} \mu_{2}+f_{2} \mu_{1}\right)^{2}+\left(f_{2} \mu_{3}+f_{3} \mu_{2}\right)^{2}+\left(f_{1} \mu_{3}+f_{3} \mu_{1}\right)^{2} \\
+2\left(f_{1} f_{2} \mu_{3}^{2}+f_{2} f_{3} \mu_{1}^{2}+f_{1} f_{3} \mu_{2}^{2}\right), \\
\gamma_{2}=\left(f_{1} \mu_{2} \mu_{3}+f_{2} \mu_{1} \mu_{3}+f_{3} \mu_{1} \mu_{2}\right) \mu_{1} \mu_{2} \mu_{3}, \\
\gamma_{3}=\delta_{T}=\left(f_{1} \mu_{2} \mu_{3}+f_{2} \mu_{1} \mu_{3}+f_{3} \mu_{1} \mu_{2}\right)^{2}, \\
\alpha_{T}=f_{1} f_{2}\left(\mu_{1}-\mu_{2}\right)^{2}+f_{1} f_{3}\left(\mu_{1}-\mu_{3}\right)^{2}+f_{2} f_{3}\left(\mu_{2}-\mu_{3}\right)^{2}, \\
\beta_{T}=\left(f_{1} \mu_{2}+f_{2} \mu_{1}\right)^{2}+\left(f_{3} \mu_{1}+f_{1} \mu_{3}\right)^{2}+\left(f_{2} \mu_{3}+f_{3} \mu_{2}\right)^{2}, \\
\gamma_{T}=f_{1} f_{2}\left(\mu_{1}-\mu_{2}\right)^{2} \mu_{3}^{2}+f_{1} f_{3}\left(\mu_{1}-\mu_{3}\right)^{2} \mu_{2}^{2}+f_{2} f_{3}\left(\mu_{2}-\mu_{3}\right)^{2} \mu_{1}^{2},
\end{gathered}
$$

where $\rho_{0}$ is the zero field resistivity, $\mu_{i}$ is the mobility of the $i$ th carrier, and $f_{i}=\left|n_{i} \mu_{i}\right| / \Sigma\left|n_{i} \mu_{i}\right|$ is the $f$ factor, with $n_{i}$ as the carrier concentration, as defined in Ref. 13. The agreement with our experimental values of $R_{H}$ was excellent as the solid lines shown in Fig. 3. FeSb ${ }_{2}$ can be described well by a two-carrier model. $\mathrm{Fe}_{1-x} \mathrm{Co}_{x} \mathrm{Sb}_{2}$ is best described within a three-carrier model since a two-carrier model cannot explain minima and maxima in $R_{H}=\rho_{x y} / H$; therefore, only threecarrier fits are shown in Figs. 3(b)-3(d). Using the obtained fitting parameters for the Hall constant, we can now solve numerically for mobilities of individual carriers and MR. We show the result in Fig. 4: The calculated orbital MR is approximately 4 orders of magnitude smaller than the observed MR for all $x$. This suggests that the conventional orbital mechanism cannot account for the large magnetoresistance in $\mathrm{Fe}_{1-x} \mathrm{Co}_{x} \mathrm{Sb}_{2}$. A similar shape of calculated and measured 

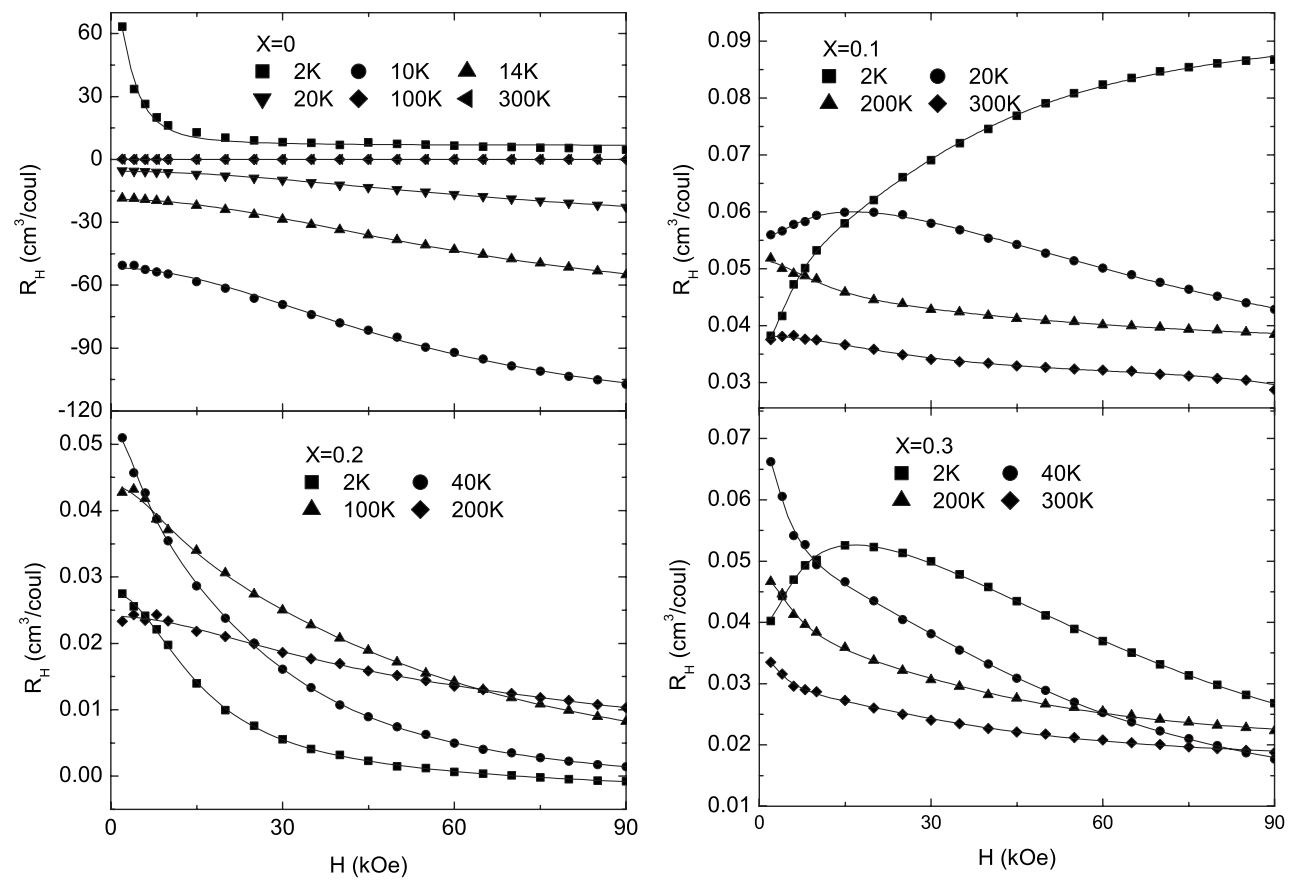

FIG. 3. (a) Hall constant $R_{H}=\rho_{x y} / H$ of $\mathrm{FeSb}_{2}$ is well described by a two-carrier model. [(b)-(d)] For $\mathrm{Fe}_{1-x} \mathrm{Co}_{x} \mathrm{Sb}_{2}(0.1 \leqslant x \leqslant 0.4)$, the fits of the two carrier model to experimental data are rather unsatisfactory since it cannot account for maxima and minima in $R_{H}(H)$. Co substitution in $\mathrm{Fe}_{1-x} \mathrm{Co}_{x} \mathrm{Sb}_{2}$ fills energy bands that are not involved in thermal excitation of carriers across the gap in $\mathrm{FeSb}_{2} . R_{H}$ shows an excellent agreement with the three-carrier model for all $x$; selected data are shown for clarity. Solid lines are fits to the multicarrier model.

MR, taken together with Fig. 3, argues in favor of the validity of the multiband electronic transport. In what follows, we show that the experimental MR is amplified by quantum interference effects.

Disordered metallic $\mathrm{Fe}_{1-x} \mathrm{Co}_{x} \mathrm{Sb}_{2}$ alloys are derived from an insulator with strong correlation effects. ${ }^{4}$ Therefore, a possible mechanism for enhanced positive MR could involve change of electronic structure induced by $\mathrm{Co}$ and quantum interference effects through the contribution of electronelectron interaction enhanced by disorder. ${ }^{15,16}$ Figure 5(a) shows qualitatively that the contribution of Coulomb interaction in the presence of random impurity scattering may be

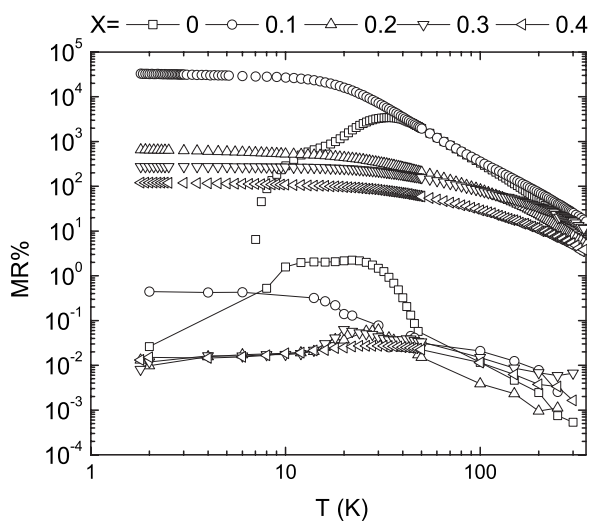

FIG. 4. Magnetoresistance calculated according to the multicarrier model for $\mathrm{Fe}_{1-x} \mathrm{Co}_{x} \mathrm{Sb}_{2}$ in $90 \mathrm{kOe}$ shown in the low plot as linked symbols, compared to the observed MR shown as the scattered symbols.
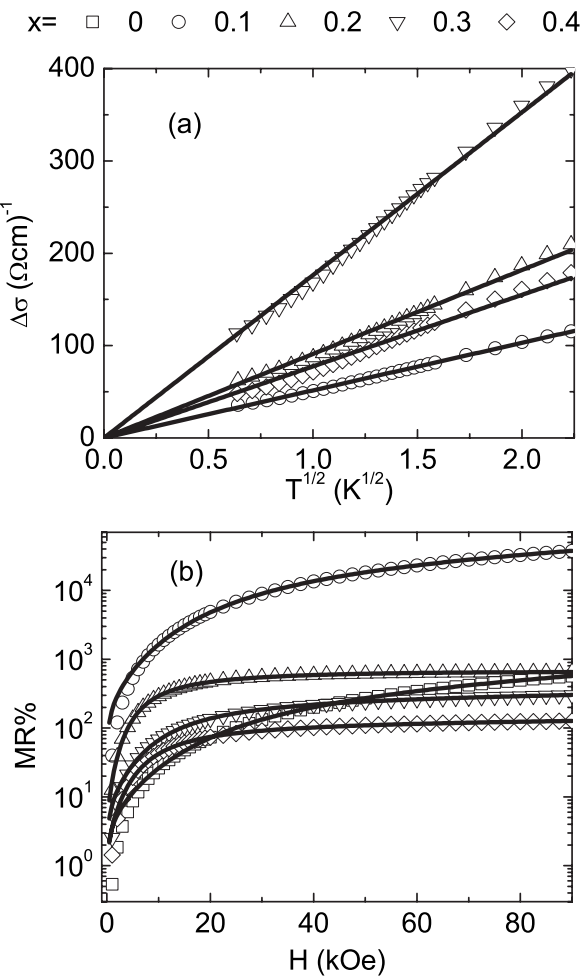

FIG. 5. (a) Magnetoconductivity of $\mathrm{Fe}_{1-x} \mathrm{Co}_{x} \mathrm{Sb}_{2}$. Conductivity change $\Delta \sigma=\sigma(H, T)-\sigma(H, 0)$, with $\sigma(H, 0)$ determined from fits of data to a $T^{1 / 2}$ dependence at $H=90 \mathrm{kOe}$. (b) Quasi-1D weak localization fits to MR at $T=1.8 \mathrm{~K}$ for $\mathrm{Fe}_{1-x} \mathrm{Co}_{x} \mathrm{Sb}_{2}$. Data for $x=0$ are shown at $T=100 \mathrm{~K}$ in the metallic quasi-1D regime of the $\hat{b}$-axis conductivity. 
TABLE I. Parameters of the fits to quantum correction of magnetoresistance. $\alpha F$ is the proportional parameter in the Coulomb interaction contribution. $L_{f}$ is the phase coherence length, $b$ is the $1 \mathrm{D}$ conduction channel width, and $c$ is the coefficient of the classical quadratic term.

\begin{tabular}{lcccc}
\hline \hline$x$ & $\alpha F$ & $\begin{array}{c}L_{f} \\
(\mathrm{~nm})\end{array}$ & $\begin{array}{c}b \\
(\mathrm{~nm})\end{array}$ & $c$ \\
\hline 0 & $1.2 \times 10^{-9}$ & 162 & 1.6 & 0.001 \\
0.1 & $6.2 \times 10^{-7}$ & 1463 & 0.2 & 0.055 \\
0.2 & $5.1 \times 10^{-9}$ & 534 & 2.8 & 0.004 \\
0.3 & $7.3 \times 10^{-9}$ & 188 & 4.0 & 0.002 \\
0.4 & $9.9 \times 10^{-9}$ & 128 & 5.1 & 0.001 \\
\hline \hline
\end{tabular}

important. More information is obtained from the magnetic field dependence of positive MR isotherms taken at $1.8 \mathrm{~K}$ [shown in Fig. 5(b)]. In the limit of strong spin-orbit scattering, the correction to quadratic MR along the high conductivity axis in $\mathrm{Fe}_{1-x} \mathrm{Co}_{x} \mathrm{Sb}_{2}$ is expressed by

$$
\begin{aligned}
\mathrm{MR}= & 1 / \rho_{0} 0.77 \alpha F\left(g \mu_{B} / k_{B}\right)^{1 / 2} H^{1 / 2} \\
& -\frac{\rho_{0} e^{2} L_{f}}{\pi \hbar b^{2}}\left[\left(1+\frac{b^{2} L_{f}^{2}}{12 L_{B}^{4}}\right)^{-1 / 2}-1\right]+c H^{2},
\end{aligned}
$$

where $L_{f}$ is the phase coherence length, $L_{B}=\sqrt{\hbar / 2 e B}$ is the magnetic length, $b$ is the width of the quasi-one-dimensional (quasi-1D) channel, and $F$ is from Hartree interaction. The first term is the contribution of the Coulomb interaction, ${ }^{17,18}$ and the second term is the contribution of the weak localization of quasi-1D model. ${ }^{19,20}$ Magnetoresistance is positive, as expected for the case of strong spin-orbit scattering in nearly magnetic conductors such as $\mathrm{Pd}$ and $\mathrm{Pt}$ alloys, and also $\mathrm{FeSb}_{2}{ }^{4,21}$ The fitting parameters are listed in Table I. As we can see, the contribution of the Coulomb interaction to MR is of the order of several percent, and the weak localization is dominant. Two inequalities justify our quasi-1D model: (1) The fitting parameters satisfy the criterion for the interfer- ence correction to be of a 1D character: $b<L_{f}$ and (2) in a field of $9 \mathrm{~T}$, the magnetic length $L_{B}$ is about $6 \mathrm{~nm}$. Therefore, $b<L_{B}$ is satisfied; otherwise, the system should behave as three dimensional. The calculated MR agrees well with the observed MR. At $T=1.8 \mathrm{~K}$, the calculated $\mathrm{MR}$ in an $H=90 \mathrm{kOe}$ field is about $34300 \%$ for $\mathrm{Fe}_{0.9} \mathrm{Co}_{0.1} \mathrm{Sb}_{2}$, as compared to the observed values of $36088 \%$. The value is derived using a measured carrier concentration $n=8.5 \times 10^{19} \mathrm{~cm}^{-3}$ and a residual resistivity $\rho_{0}=1.0$ $\times 10^{-5} \Omega \mathrm{cm}$ of $\mathrm{Fe}_{0.9} \mathrm{Co}_{0.1} \mathrm{Sb}_{2}$, and $L_{f} \simeq 240 L_{B}$. The width of the quasi-1D channel $b$ is of the order of the unit cell. Quasi-1D localization effects are observed in pure $\mathrm{FeSb}_{2}$ at $100 \mathrm{~K}$ [Fig. 5(b)]. It implies that the electronic transport is dominated by the singular corrections at the density of states at unusually high temperatures, apparently without the usual cutoff by thermal effects, probably due to the high spin-orbit scattering rate $1 / \tau s .{ }^{21} \mathrm{~A}$ similar situation has been observed in $\mathrm{Ta}_{4} \mathrm{Te}_{4} \mathrm{Si}$ at $15 \mathrm{~K}$ (Ref. 22) and Si nanowires at $27 \mathrm{~K}^{23}$

In conclusion, we have shown CMR effect in doped nearly magnetic semiconductor $\mathrm{FeSb}_{2}$. The application of magnetic fields up to $350 \mathrm{kOe}$ results in $103100 \%$ increase in the resistivity at $1.8 \mathrm{~K}$ and $124 \%$ increase at room temperature. Our results suggest a contribution of multiple electronic bands to electrical conduction. Quantum interference arising from Coulomb interactions and weak localization in the presence of strong spin-orbit scattering is the dominant mechanism of CMR.

We thank Maxim Dzero, Sergey Bud'ko, Zachary Fisk, and T. Maurice Rice for useful communication. This work was carried out at the Brookhaven National Laboratory, which is operated for the U.S. Department of Energy by Brookhaven Science Associates (DE-Ac02-98CH10886). A portion of this work was performed at the National High Magnetic Field Laboratory, which is supported by NSF Cooperative Agreement No. DMR-0084173, by the State of Florida, and by the U.S. Department of Energy. This work was also supported in part by the National Science Foundation Grant No. DMR-0547938 (V.F.M.).
*Present address: Nature Publishing Group, London, UK.

†resent address: Department of Chemistry and Biochemistry, University of South Carolina, Columbia, SC 29208, USA.

Present address: Institut für Festkörperforschung, Forschungzentrum Jülich GmbH, D-52425 Jülich, Germany.

${ }^{1}$ V. I. Anisimov, R. Hlubina, M. A. Korotin, V. V. Mazurenko, T. M. Rice, A. O. Shorikov, and M. Sigrist, Phys. Rev. Lett. 89, 257203 (2002).

${ }^{2}$ C. Petrovic, J. W. Kim, S. L. Bud'ko, A. I. Goldman, P. C. Canfield, W. Choe, and G. J. Miller, Phys. Rev. B 67, 155205 (2003).

${ }^{3}$ C. Petrovic, Y. Lee, T. Vogt, N. Dj. Lazarov, S. L. Bud'ko, and P. C. Canfield, Phys. Rev. B 72, 045103 (2005).

${ }^{4}$ A. V. Lukoyanov, V. V. Mazurenko, V. I. Anisimov, M. Sigrist, and T. M. Rice, Eur. Phys. J. B 53, 205 (2006).

${ }^{5}$ Rongwei Hu, V. F. Mitrovic, and C. Petrovic, Phys. Rev. B 74,
195130 (2006)

${ }^{6}$ A. Perucchi, L. Degiorgi, Rongwei Hu, C. Petrovic, and V. F. Mitrovic, Eur. Phys. J. B 54, 175 (2006).

${ }^{7}$ S. Yeo, S. Nakatsuji, A. D. Bianchi, P. Schlottmann, Z. Fisk, L. Balicas, P. A. Stampe, and R. J. Kennedy, Phys. Rev. Lett. 91, 046401 (2003).

${ }^{8}$ N. Manyala, Y. Sidis, J. F. DiTusa, G. Aeppli, D. P. Young, and Z. Fisk, Nature (London) 404, 581 (2000).

${ }^{9}$ Myron B. Salamon and Marcelo Jaime, Rev. Mod. Phys. 73, 583 (2001).

${ }^{10}$ J. S. Dodge, C. P. Weber, J. Corson, J. Orenstein, Z. Schlesinger, J. W. Reiner, and M. R. Beasley, Phys. Rev. Lett. 85, 4932 (2000).

${ }^{11}$ R. C. O'Handley, in The Hall Effect and Its Application, edited by C. L. Chien and C. R. Westgate (Plenum, New York, 1980), p. 417. 
${ }^{12}$ E. Finkman and Y. Nemirovsky, J. Appl. Phys. 53, 1052 (1982).

${ }^{13}$ J. S. Kim, J. Appl. Phys. 86, 3187 (1999).

${ }^{14}$ J. S. Kim, J. Appl. Phys. 84, 292 (1998).

${ }^{15}$ Patrick A. Lee and T. V. Ramakrishnan, Rev. Mod. Phys. 57, 287 (2005).

${ }^{16}$ J. S. Dugdale, The Electrical Properties of Disordered Metals (Cambridge University Press, New York, 1995).

${ }^{17}$ S. Morita, Y. Isawa, T. Fukase, S. Ishida, Y. Koike, Y. Takeuti, and N. Mikoshiba, Phys. Rev. B 25, 5570 (1982).

${ }^{18}$ P. A. Lee and T. V. Ramakrishnan, Phys. Rev. B 26, 4009 (1982).
${ }^{19}$ B. L. Al'tshuler and A. G. Aronov, JETP Lett. 33, 499 (1981).

${ }^{20}$ A. I. Yakimov and A. V. Dvurechenskii, JETP Lett. 69, 202 (1999).

${ }^{21}$ A. J. Millis and P. A. Lee, Phys. Rev. B 30, 6170 (1984).

${ }^{22}$ A. Stolovits, A. Sherman, K. Ahn, and R. K. Kremer, Phys. Rev. B 62, 10565 (2000).

${ }^{23}$ J. F. Dayen, A. Rumyantseva, C. Ciornei, T. L. Wade, J. E. Wegrowe, Pribat, and C. Sorin Cojocaru, Appl. Phys. Lett. 90, 173110 (2007). 